\title{
Genic and nongenic contributions to natural variation of quantitative traits in maize
}

\author{
Xianran Li, ${ }^{1}$ Chengsong Zhu, ${ }^{1}$ Cheng-Ting Yeh, ${ }^{2}$ Wei Wu, ${ }^{2}$ Elizabeth M. Takacs, ${ }^{3}$ \\ Katherine A. Petsch, ${ }^{4}$ Feng Tian, ${ }^{5}$ Guihua Bai, ${ }^{1,6}$ Edward S. Buckler, ${ }^{5,6}$ Gary J. Muehlbauer, ${ }^{7}$ \\ Marja C.P. Timmermans, ${ }^{4}$ Michael J. Scanlon, ${ }^{3}$ Patrick S. Schnable, ${ }^{2,8}$ and Jianming $\mathrm{Yu}^{1,8}$ \\ ${ }^{7}$ Department of Agronomy, Kansas State University, Manhattan, Kansas 66506, USA; ${ }^{2}$ Center for Plant Genomics and Department \\ of Agronomy, lowa State University, Ames, lowa 5001 1, USA; ${ }^{3}$ Department of Plant Biology, Cornell University, Ithaca, New York 14853 , \\ USA; ${ }^{4}$ Cold Spring Harbor Laboratory, Cold Spring Harbor, New York 11724, USA; ${ }^{5}$ Institute for Genomic Diversity, Cornell University, \\ Ithaca, New York 14853, USA; ${ }^{6}$ United States Department of Agriculture-Agricultural Research Service (USDA-ARS), Manhattan, Kansas \\ 66506, USA; ${ }^{7}$ Department of Agronomy and Plant Genetics, University of Minnesota, St. Paul, Minnesota 55108, USA
}

\begin{abstract}
The complex genomes of many economically important crops present tremendous challenges to understand the genetic control of many quantitative traits with great importance in crop production, adaptation, and evolution. Advances in genomic technology need to be integrated with strategic genetic design and novel perspectives to break new ground. Complementary to individual-gene-targeted research, which remains challenging, a global assessment of the genomic distribution of trait-associated SNPs (TASs) discovered from genome scans of quantitative traits can provide insights into the genetic architecture and contribute to the design of future studies. Here we report the first systematic tabulation of the relative contribution of different genomic regions to quantitative trait variation in maize. We found that TASs were enriched in the nongenic regions, particularly within a 5-kb window upstream of genes, which highlights the importance of polymorphisms regulating gene expression in shaping the natural variation. Consistent with these findings, TASs collectively explained $44 \%-59 \%$ of the total phenotypic variation across maize quantitative traits, and on average, $79 \%$ of the explained variation could be attributed to TASs located in genes or within $5 \mathrm{~kb}$ upstream of genes, which together comprise only $13 \%$ of the genome. Our findings suggest that efficient, cost-effective genome-wide association studies (GWAS) in species with complex genomes can focus on genic and promoter regions.
\end{abstract}

[Supplemental material is available for this article.]

Cloning of individual large-effect genes underlying qualitative and quantitative traits has provided some insights into the genetic control of trait variation. These studies have most frequently implicated nucleotide polymorphisms in genic regions as being causative (Doebley et al. 2006; Miura et al. 2011); however, generalizing from these results may not be appropriate because of ascertainment bias, e.g., preference given to genic regions during the efforts in gene cloning. In addition, it remains challenging to identify, validate, and characterize genes underlying modest-tosmall-effect quantitative trait loci (QTLs), which are common contributors to quantitative traits in crops with complex genomes. With these challenges, a different approach is to identify what part of a complex genome can be prioritized. Intuitively, this is known from mutational dissection of "qualitative phenotypes," but a global assessment for "quantitative traits" is lacking and the relative importance of genic and nongenic portions of the genome has significant bearings on further biological research and crop improvement.

By identifying trait-associated SNPs (TASs), genome-wide association studies (GWASs) can enhance our understanding of the genetic architecture (Meyer et al. 2008; Chang et al. 2009;

\footnotetext{
${ }^{8}$ Corresponding authors Email jyu@ksu.edu Email schnable@iastate.edu

Article published online before print. Article, supplemental material, and publication date are at http://www.genome.org/cgi/doi/10.1101/gr.140277.112. Freely available online through the Genome Research Open Access option.
}

Teslovich et al. 2010). For example, a survey of 531 human TASs found that most are located in noncoding regions (43\% from nongenic regions and $45 \%$ from introns), suggesting that the search for functional polymorphisms should extend beyond coding regions (Hindorff et al. 2009). Indeed, some recent individualgene studies have suggested that functional nongenic polymorphisms can also contribute to the variation associated with quantitative traits in plants (Frary et al. 2000; Stam et al. 2002; Ashikari et al. 2005; Clark et al. 2006; Salvi et al. 2007). However, previous GWASs in plants focused on single SNP testing or multiple regression (Atwell et al. 2010; Huang et al. 2010; Tian et al. 2011) and did not address this critical issue. Hence, a systematic evaluation of TASs in plants can help to answer several important questions: (1) What are the overall contributions of genetic polymorphisms (i.e., SNPs) in explaining the phenotypic variation of quantitative traits; (2) what are the relative contributions of genic and nongenic polymorphisms; and (3) what is the distribution of maize TASs across different genomic annotation sets (e.g., promoter, intron, or coding region)?

Here we report genome scans of five quantitative traits with SNPs identified by two complementary next-generation sequencing strategies to identify the underlying TASs, the genomic distribution of these TASs, and the relative contributions of genic and nongenic TASs to the phenotypic variation. We found that genic and nongenic TASs contribute approximately equally to the phenotypic variation of maize quantitative traits. But the distributions of maize TASs in specific annotation sets differed. Specifically, nonsynonymous SNPs are underrepresented among TASs for 
maize quantitative traits, suggesting that regulatory variation plays an important role in phenotypic variation. Our results suggest that genotyping methods designed to discover SNPs in genes and their upstream regions can be an economical approach for detecting genome-wide association signals in future GWAS scans of quantitative traits in crops with complex genomes.

\section{Results}

To be consistent with the GWAS literature (Hindorff et al. 2009), a genic region in this study is defined as between the transcription start site (TSS) and the end of 3' UTR. Toward this end, we first conducted RNA-seq to identify gene-enriched SNPs. A targeteddissection genome scan method was implemented to identify the TASs for five maize quantitative traits (leaf length, leaf width, upper leaf angle, days to anthesis, and days to silking) from 1 million SNPs merged from the RNA-seq data and the previously defined set of maize HapMap SNPs (Gore et al. 2009). We then systematically characterized the genomic distribution and genetic features of the discovered TASs across regions and traits. Two annotations sets (high stringent filtered gene set [FGS] and low stringent working gene set [WGS]) have been released for maize genome (Schnable et al. 2009). Because the results obtained from the analysis of the FGS and WGS were similar, for the simplicity of discussion only, the FGS analysis results are presented in the text, but the results from the WGS analysis are included in the Supplemental Material.

\section{RNA-seq and SNPs for GWAS}

RNA was extracted from shoot apices, which includes the shoot apical meristem (SAM) and up to five young leaf primordia, of 2-wk-old seedlings from each of the nested association mapping (NAM) founders (Methods) and used to conduct RNA-seq. To discover SNPs, the RNA-seq reads were aligned to the B73 maize reference genome sequence (Schnable et al. 2009). This tissue and stage of development were selected because we had previously shown that a substantial percentage of genes is expressed in the SAM and leaf primordia at this stage of development (Emrich et al. 2007) and because we were interested in testing whether SNPs identified from genes expressed at this stage of development are enriched for genes related to leaf architecture traits versus flowering-time traits.

Nearly 1 million SNPs $(N=942,793)$ were identified from approximately 600 million 76-bp RNA-seq reads from the 26 inbred founders (not including B73) of the NAM population. Of all discovered SNPs, only 289,461 that could be called with high confidence (Methods) in $>81 \%$ of the NAM founders (i.e., more than 22/27) were used for GWASs (Table 1). As expected based on their mode of discovery from RNA-seq reads, most of these SNPs were located within annotated genes. Of the 289,461 SNPs, $87 \%$ were located within 15,097 of the 32,540 FGS genes (46\%).
Similarly, we retained 774,754 HapMap (Gore et al. 2009) SNPs using the same threshold for missing SNP genotypes among the NAM founders (i.e., >22/27). About half (49\%) of the HapMap SNPs resided within 25,738 genes (79\% of the FGS), and $85 \%$ of these genes were expressed in leaves (Li et al. 2010). In total, after merging the two data sets, just over 1 million (1.01 million) SNPs, of which $58 \%$ were located within 26,382 (81\%) FGS genes, were available for GWAS (Table 1; Supplemental Fig. S1). These SNPs were projected from the NAM founders to the approximately 5000 recombinant inbred lines (RILs) based on the previous results from genotyping the NAM population with 1106 tagging SNPs (tSNP; Methods) (McMullen et al. 2009; Tian et al. 2011).

\section{GWAS of quantitative traits}

Genome scans using the 1.01 million merged SNPs identified TASs underlying five quantitative traits (Supplemental Fig. S2). This was accomplished using a two-stage scan method made possible by the genetic design of NAM (Yu et al. 2008). The first stage was a lowresolution mapping using 1106 tSNPs. This analysis identified 164 QTLs for the five quantitative traits; 44 of the 164 QTL regions were colocalized by the adjacent or common tSNPs. The 164 target QTL regions covered $\sim 67 \%$ of the maize genome, with an average size of $400 \mathrm{Mb}$ for each trait. In the second stage of the genome scan, the 1.01 million SNPs, which had been projected from the NAM founders to the RILs, were tested for associations with the five traits. Target regions were defined as the three tSNPs to the left and the three tSNPs right of the tSNP most strongly associated with each QTL in the first genome-wide QTL scan (Supplemental Fig. S3). For each target region, on average, 7319 SNPs located among the seven tSNPs were analyzed in this step. In terms of answering the critical aforementioned questions, this targeted scan is significantly different (see Discussion) from previous analysis, in which an individual SNP search across a chromosome is conducted. Here, only the target regions were scanned for TASs to avoid false discoveries that might otherwise be introduced from nearby regions. Genome-wide polygenic effects were controlled by including other QTLs in the model during the scan. To control for multiple testing, a minimum false-discovery rate, Q-value (Storey and Tibshirani 2003), was calculated for each TAS. The vast majority of TASs ( $85 \%)$ had a $Q$-value from $1.0 \times 10^{-4}$ to $4.7 \times 10^{-105}$ (Supplemental Table S1). After the genome scan, 40 out of 44 of the colocalized QTL regions were dissected to independent TASs, demonstrating the high resolving power of this GWAS (Supplemental Fig. S2). The common TASs detected for other four colocalized QTL regions were not unexpected because they were detected for two flowering-time traits that have a higher phenotypic correlation than other pairs of traits. RNAseq SNPs yielded the strongest signals in 16 of the 164 dissected regions (Supplemental Fig. S1). Meanwhile, of the genes implicated by the highest signals from HapMap SNPs, 58\% also har-

Table 1. Distribution of maize SNPs across different genomic annotation sets

\begin{tabular}{|c|c|c|c|c|c|c|c|c|}
\hline Data set & Nongenic & $\begin{array}{l}\text { Promoter } \\
5 \mathrm{~kb}\end{array}$ & $\begin{array}{l}\text { Promoter } \\
1 \text { kb }\end{array}$ & Intron & $5^{\prime}$ UTR & 3' UTR & Syn & Nsy \\
\hline HapMap & $394,160(51 \%)$ & $149,018(19 \%)$ & $77,513(10 \%)$ & $109,912(14 \%)$ & $47,312(6 \%)$ & $33,264(4 \%)$ & $98,434(13 \%)$ & $83,271(11 \%)$ \\
\hline RNA-seq & $36,416(13 \%)$ & $8,863(3 \%)$ & $2,588(1 \%)$ & $5,663(2 \%)$ & $15,597(5 \%)$ & $62,983(22 \%)$ & $101,907(35 \%)$ & $66,355(23 \%)$ \\
\hline Merged & $424,703(42 \%)$ & $156,197(16 \%)$ & $79,448(8 \%)$ & $114,784(11 \%)$ & $57,814(6 \%)$ & $88,152(9 \%)$ & $176,435(18 \%)$ & $134,484(14 \%)$ \\
\hline
\end{tabular}

Nongenic includes promoter $5 \mathrm{~kb}$, which in turn includes promoter $1 \mathrm{~kb}$. (UTR) Untranslated region; (Syn) synonymous SNPs; (Nsy) nonsynonymous SNPs. 
bored RNA-seq SNPs with strong association signals, demonstrating the value of using RNA-seq SNPs for GWAS.

\section{Distribution of TASs}

Only $\sim 6 \%$ of the maize genome is genic (Schnable et al. 2009). Knowledge of the relative proportion of TASs located in genic and nongenic regions would shed light on the relative contributions of these two regions to quantitative trait variation. To test whether the final identification of TASs was context independent (i.e., to confirm that identifying a nongenic TAS was not simply due to a high proportion of nongenic SNPs within the starting SNP set), the 164 dissected QTL regions were also separately scanned for TASs using only the RNA-seq SNPs or only the HapMap SNPs in addition to the merged SNP data set (Fig. 1; Supplemental Fig. S4). For each analysis and for each QTL region, we identified the TAS with the highest signal and classified it as genic or nongenic. Summarizing the results across all 164 dissected QTL regions provided a genome-wide picture of the relative distributions of genic and nongenic TASs (Fig. 1). From the merged SNP set, nongenic TASs were identified for $46 \%$ of the dissected regions even though only a few (6\%) regions were classified as predominantly nongenic (i.e., containing a higher proportion of nongenic than genic SNPs). From the gene-enriched RNA-seq SNP set, nongenic TASs were detected for $16 \%$ of dissected QTL regions. When only the HapMap SNP set was analyzed, however, the classification of TASs obtained was not independent of the classification of the regions. A similar pattern was observed when the top five highest associated SNPs within each region were tabulated as TASs (Supplemental Table S2). Linkage disequilibrium analysis among the top five highest associated SNPs suggested that the identified genomic regions and the TASs are well supported (Fig. 2). For the majority of these QTLs, the strongest association signals were concentrated within a window of 500-1000 bp around the TASs. Moreover, genic or nongenic TASs were equally likely to be identified within the QTL regions with either large or small genetic effects, as shown by the wellmixed pattern of TASs when the QTL effects were sorted in descending order for each trait (Fig. 1; Supplemental Fig. S4; Supplemental Table S2). Because very few shared TASs (3\%) were found among traits and because the proportion of nongenic TASs was consistent across traits, our findings from these five traits may represent a general feature of TASs underlying other quantitative traits in plants, or at least cross-pollinated crops that have been subjected to intense selection.

\section{TASs in different genomic annotation sets}

We further evaluated the distribution of TASs by testing for their enrichment in eight sets of genomic annotations (nongenic region, promoter $5 \mathrm{~kb}$, promoter $1 \mathrm{~kb}, 5^{\prime} \mathrm{UTR}$, synonymous site, nonsynonymous site, intron, and 3' UTR) (Table 1). Because promoters are typically located upstream of TSSs, for the purposes of this analysis, we defined $1 \mathrm{~kb}$ of the TSS as the promoter $1 \mathrm{~kb}$, and likewise $5 \mathrm{~kb}$ upstream of the TSS as promoter $5 \mathrm{~kb}$, respectively. Three annotation sets (nongenic region, promoter $5 \mathrm{~kb}$, and nonsynonymous) exhibited significantly different proportions of genic or nongenic TASs from the tested SNPs (Fig. 3). TASs were overrepresented in nongenic $(P=$ $0.026)$ and promoter $5 \mathrm{~kb}$ regions $(P=$ 0.041) (Supplemental Fig. S5). Although $13 \%$ of the $1.01 \mathrm{M}$ SNPs were nonsynonymous, only $4 \%$ of TASs were nonsynonymous SNPs, indicating that nonsynonymous polymorphisms are underrepresented among maize TASs $(P=$ $2.7 \times 10^{-6}$ ).

Of 27 TASs within the promoter $5 \mathrm{~kb}$ set, 19 were located upstream of genes for which duplication or single copy has been characterized in a maize genome duplication study (Schnable et al. 2011). Interestingly, 12 of these TASs are located in the upstream of genes with duplicated copies (4507), and seven are located in the upstream of genes without duplication (9867).

\section{Variation explained by TASs}

In the NAM population, $74 \%-89 \%$ of the phenotypic variation for each of the five analyzed quantitative traits is explained by $28 \%-37 \%$ QTLs. Similar to findings from other complex trait studies, only a small fraction of the variation can be
Figure 1. Genic or nongenic TASs for each dissected QTL region and the proportion of genic SNPs among all tested in the region. Genic region is defined as from the transcription start site to the end of $3^{\prime}$ UTR. With the merged data set, the probability of a TAS being genic or nongenic is significantly independent of whether more genic or nongenic SNPs in the target region are tested $(P<0.05)$ for all five traits. Each row represents a single QTL region for the indicated trait. Genic and nongenic TASs are equally likely to be identified for QTLs with large or small effects, which are sorted in descending order within each trait. (DTS) Days to silking.

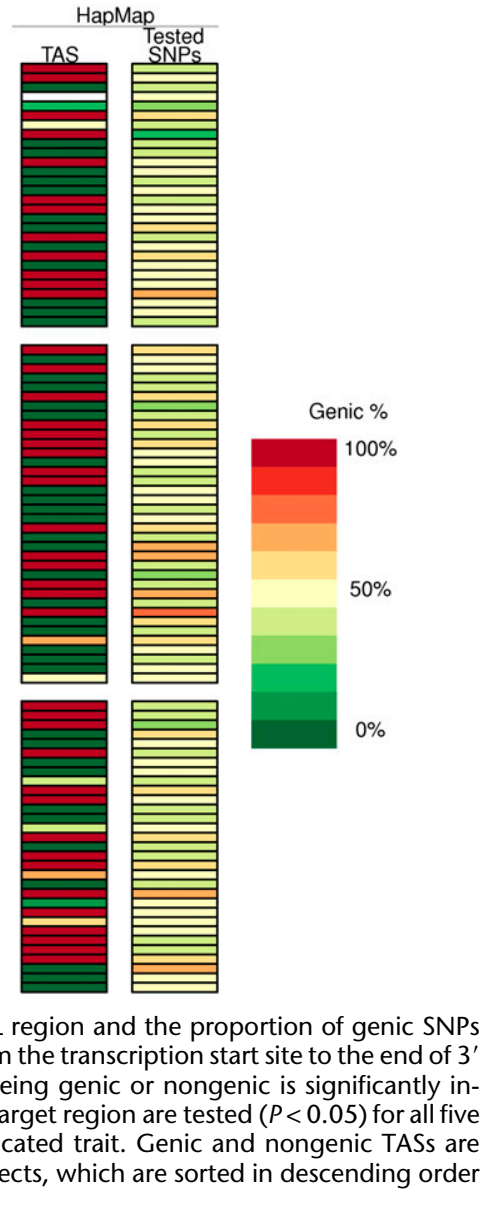

\section{Genome Research}

www.genome.org 


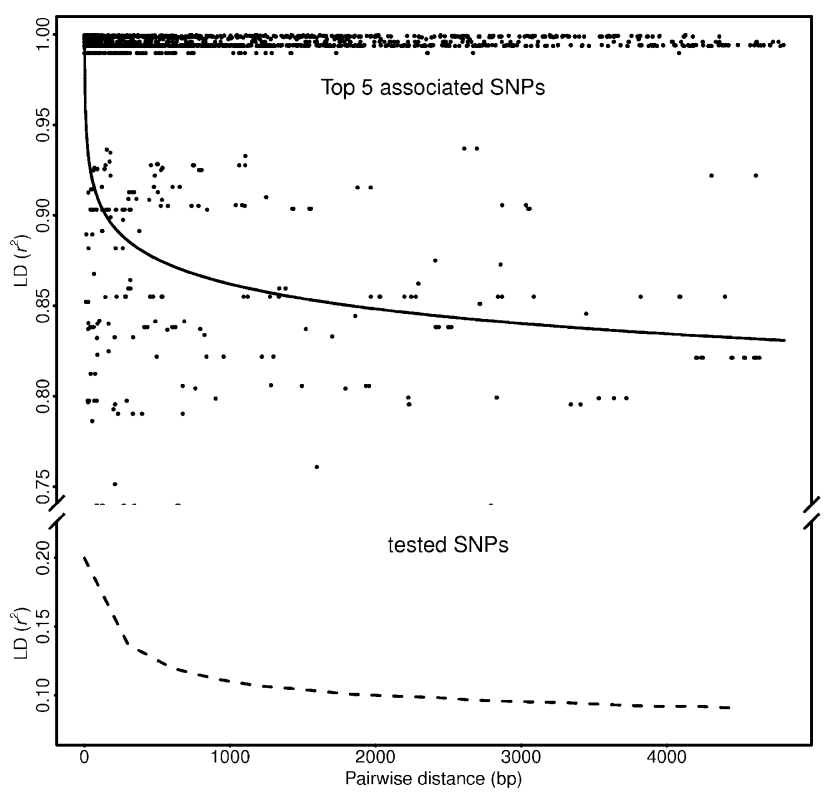

Figure 2. Linkage disequilibrium (LD) among the top five associated SNPs within each target region and the LD among all tested SNPs. A strong LD among top five associated SNPs and a plateau beyond $500-1,000$ bp indicate that genomic regions signaled by TASs are well supported.

captured by individual TASs; however, these TASs collectively explained $44 \%-53 \%$ of the total phenotypic variation (Fig. 4). And this reduction in the amount of explained phenotypic variation by TASs compared with QTLs (equivalent to $56 \%-67 \%$ of heritability by TASs) is much smaller than what has been reported in human GWAS summaries, which is typically $<10 \%$ (Manolio et al. 2009). As expected from the genetic design, the contribution from an individual TAS to the overall phenotypic variation is determined by the allele frequency and genetic effect (Fig. 5). A relatively large contribution from a TAS required a balanced allele frequency (i.e., approaching to 0.5), a modest to large genetic effect size, or both.

Upon partitioning TASs into genic or nongenic groups, $46 \%-$ $63 \%$ of the explained variation (i.e., $21 \%-35 \%$ of the total phenotypic variation) is attributed to genic TASs across the five traits. Because maize TASs were found to be overrepresented in upstream promoter regions, we also examined the expanded genic region (gene $+1-\mathrm{kb}$ or 5 -kb upstream region). By including upstream regions, the percentage of explained variation increased to $53 \%-$ $73 \%$ (genes $+1 \mathrm{~kb}$ ) or $67 \%-91 \%$ (genes $+5 \mathrm{~kb}$ ) (Fig. 4). Taken together, while the larger of the expanded genic region accounts for only $13 \%$ of the maize genome, they comprise $71 \%$ (by count) of the identified TASs and contribute to $79 \%$ (by variation explained) of the phenotypic variation captured by all TASs.

\section{Candidate genes implicated by TASs}

A variety of gene ontology (GO) terms were overrepresented among the TAS-implicated candidate genes, suggesting that complex networks shape these traits (Supplemental Table S3). For example, the terms "response to hormone stimulus," "protein transporter activity," and "regulation of transcription, DNAdependent" were among the most significantly overrepresented. Two genic SNPs (one intronic and one in the coding region) within liguleless2 ( $\lg 2)$, which is required to form the ligule/auricle hinge between leaf blade and sheath (Harper and Freeling 1996; Walsh et al. 1998), had the highest association value with upper leaf angle. This result differs from a previous analysis (Tian et al. 2011), in which a SNP downstream from $\lg 2$ was identified, although the association signals at all three sites were among the strongest in both analyses. Meanwhile, a TAS for leaf width were located $95 \mathrm{~kb}$ upstream of rough sheath1 (rs1), which is expressed in the base of initiating leaves and young leaf primordial (Schneeberger et al. 1995). Importantly, no other predicted maize genes are located between this TAS and the rs1 gene. Other candidate genes implicated by TASs included zea agamous 5 (Mena et al. 1995) and a YABBY transcription factor (GRMZM2G102218) for days to silking. Two AP2 domain proteins (GRMZM2G129777 and GRMZM2G421033) showed associations for leaf length and days to silking, respectively (Supplemental Table S1). This maize YABBY transcription factor is a homolog of CRABS CLAW in Arabidopsis, which has been shown to function in carpel and nectary development (Lee et al. 2005)

\section{Expression analysis}

Investigation of our new expression profile (Methods) and published transcriptome data ( $\mathrm{Li}$ et al. 2010) revealed that almost all TAS-implicated candidate genes were dynamically expressed among 10 tissues (four embryo developmental stages, two types of meristems, and four leaf gradient zones) (Supplemental Fig. S6). Indeed, the expression level of the leaf length-associated AP2 gene was higher than another AP2 gene related to flowering time (Supplemental Fig. S7). On average, TAS-implicated candidate genes associated with leaf width had higher expression levels than other genes that were expressed in leaves (Supplemental Table S4).

To provide further functional analysis of the TASs, we examined the expression patterns of TAS-implicated candidate genes in an RNA-seq comparison between a leafbladeless1 (lbl1) mutant and its wild-type controls. The $l b l 1$ mutant affects a variety of leaf developmental processes (Timmermans et al. 1998; Nogueira et al. 2007). The finding that three candidate genes (GRMZM2G047129 for leaf length; GRMZM2G083812 and GRMZM2G417843 for leaf width) were differentially expressed between $l b l 1$ mutant and wild type under one or both backgrounds (Supplemental Fig. S8) provides additional functional support for the relevant TASs.

\section{Discussion}

Maize, with extensive morphological variation and genetic diversity, has been exploited as a model species in studies of quantitative and population genetics, selection theory, domestication, breeding methods, and molecular genetics. Recent maize research in association mapping (Yu et al. 2006; Zhang et al. 2010), NAM (Yu et al. 2008; Buckler et al. 2009), genome-wide selection (Bernardo and Yu 2007; Riedelsheimer et al. 2012), and NAMGWAS scan (Tian et al. 2011) represented some new contributions to complex trait dissection and selection in plants, many of which are facing similar challenges. The maize NAM population consists of 5000 RILs derived from crossing 26 diverse founders to a common parent, B73. The NAM design combines the merits of both linkage and linkage disequilibrium mapping to detect molecular polymorphisms underlying quantitative traits (Yu et al. 2008; Buckler et al. 2009), and represents a logical framework for 
A

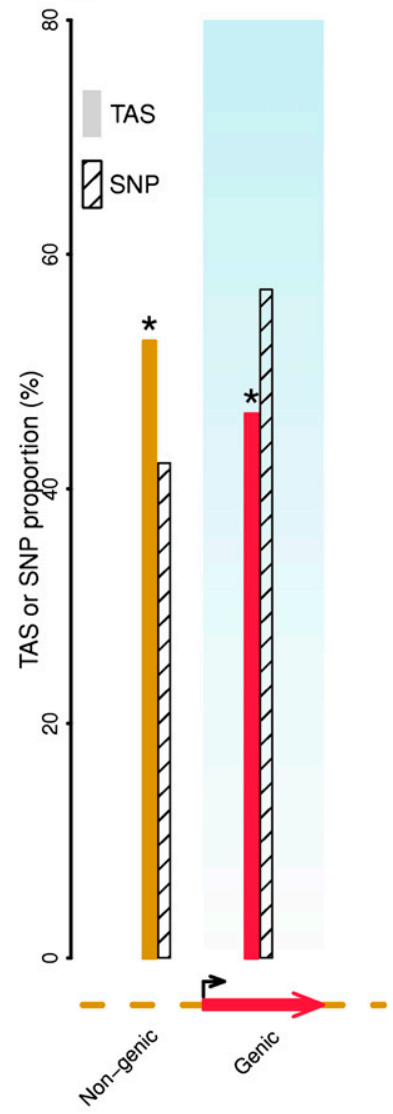

B

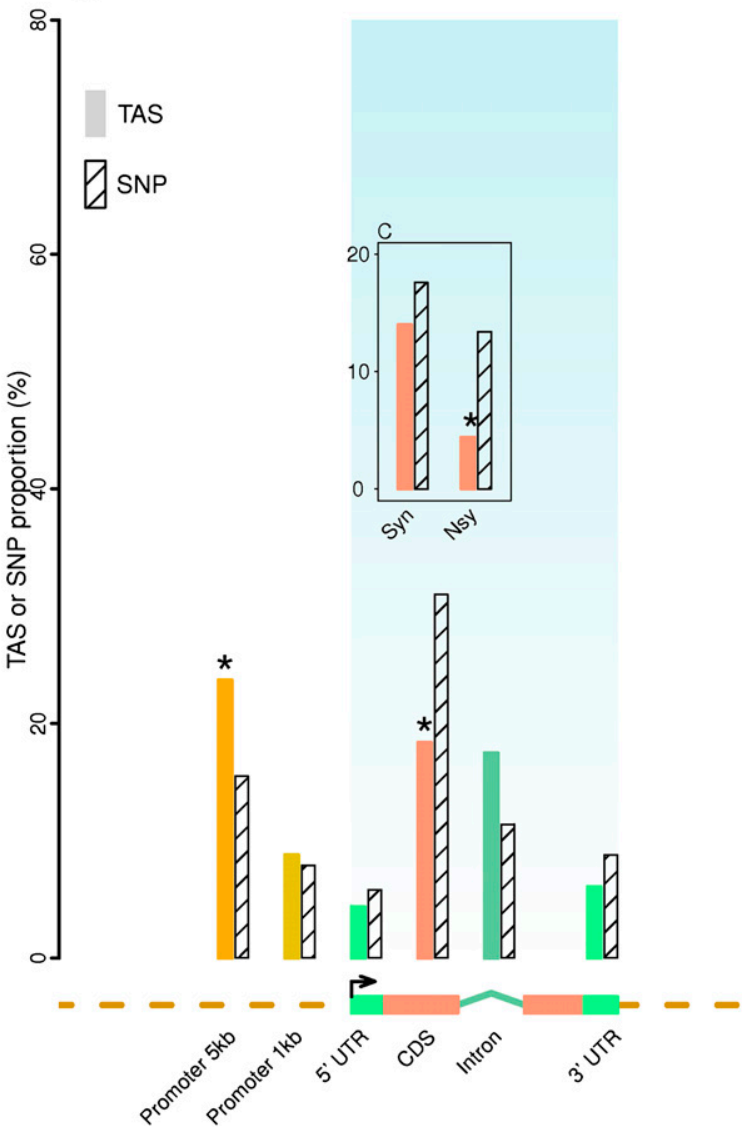

Figure 3. Distribution of TASs and tested SNPs for five quantitative traits across genomic annotation sets. (A) Nongenic versus genic (genic region is defined as from the transcription start site to the end of 3' UTR); (B) different annotation sets; and (C) synonymous versus nonsynonymous. Genic plus 5-kb upstream regions comprise only $13 \%$ of the maize genome but account for $71 \%$ of TASs. Nongenic and promoter $5 \mathrm{~kb}$ regions are overrepresented among TASs. The nonsynonymous set is underrepresented among TASs. Note that the nongenic region includes promoter $5 \mathrm{~kb}$, which in turn includes promoter $1 \mathrm{~kb}$, and the genic region includes the untranslated region (UTR), coding region (CDS), and intron. Stars denote that the proportion of TASs from the annotation set significantly differs from that of tested SNPs. (Arrow) Transcription start site.

conducting genome scans of multiple quantitative traits to answer the aforementioned questions.

\section{New angle into more important questions}

In the current study, GWAS scans of five quantitative traits (three leaf and two flowering-time traits) were conducted to estimate the relative contributions of genic and nongenic genetic variants to phenotypic variation. Instead of searching for signals on a chromosome base (Tian et al. 2011), the new targeteddissection method minimized the influence of other TAS-containing regions on the search. More importantly, this method also allowed us to tabulate the TAS findings across regions to systematically address the contribution of genic and nongenic polymorphisms to quantitative traits, which was not possible with the previous analysis. Specifically, two complementary angles were presented: number of TASs in each class, and the proportion of phenotypic variation explained by TASs in each class. Unlike a previous analysis (Tian et al. 2011), the current study included SNPs derived from the analysis of RNA-seq reads to enable a valid comparison of the relative contributions of genic versus nongenic variants, and included the analyses of two nonleaf traits (i.e., flowering time) to enable broader inferences.

\section{Value of RNA-seq for SNP discovery}

RNA-seq has been proposed as a fast and inexpensive genotyping method (Barbazuk et al. 2007; Cloonan et al. 2008; Cirulli et al. 2010). Considering that the total number of HapMap SNPs is three times that of the RNA-seq SNP set, a direct comparison between the two genotyping approaches for association tests is inappropriate; however, we demonstrated the potential of using RNA-seq to discover SNPs that are suitable for GWAS. A total of 16 of the TASs were identified from the RNA-seq SNP set, and $58 \%$ of the genes captured by the HapMap TASs also harbored strongly associated RNA-seq SNPs. Because there is much overlap between genes expressed in shoot apices and other tissues (Sekhon et al. 2011), SNPs discovered from RNAseq conducted on RNA isolated from shoot apices can be used to map a variety of complex traits. In our study, of these 16 TASs, nine were identified for the two flowering-time traits and seven for the three leaf architecture traits. More importantly, having RNA-seq SNPs enabled us to compare genome scan results with different proportions of genic SNPs (RNA-seq, HapMap, and merged). These comparisons diminished the influence of the distribution of SNPs on TASs (i.e., ascertainment bias), which therefore enabled us to evaluate the relative contributions of genic and nongenic polymorphisms on phenotypic variation of quantitative traits.

\section{Distribution of maize TASs and candidate genes}

A long-standing question in evolutionary biology is the nature of the genetic variation that controls variation in quantitative traits. That is, is the variation mainly driven by differences in protein sequence or by differences in gene expression patterns (Clark et al. 2006; Doebley et al. 2006; Alonso-Blanco et al. 2009)? In human GWASs, nonsynonymous SNPs are overrepresented among TASs (Hindorff et al. 2009), which is consistent with a major role for alterations in protein sequences (Stenson et al. 2009). In contrast, our data demonstrate that among maize TAS, nonsynonymous sites are significantly underrepresented. This is true regardless of whether we analyze the FGS (which is predicted to include few false-positive genes, but to be missing about one-third of genes) or the WGS (which is expected to include all of maize gene space plus an estimated approximately 50,000 nongenic sequences) (Supplemental Figs. S9-S11). Interestingly, recent research has dem- 

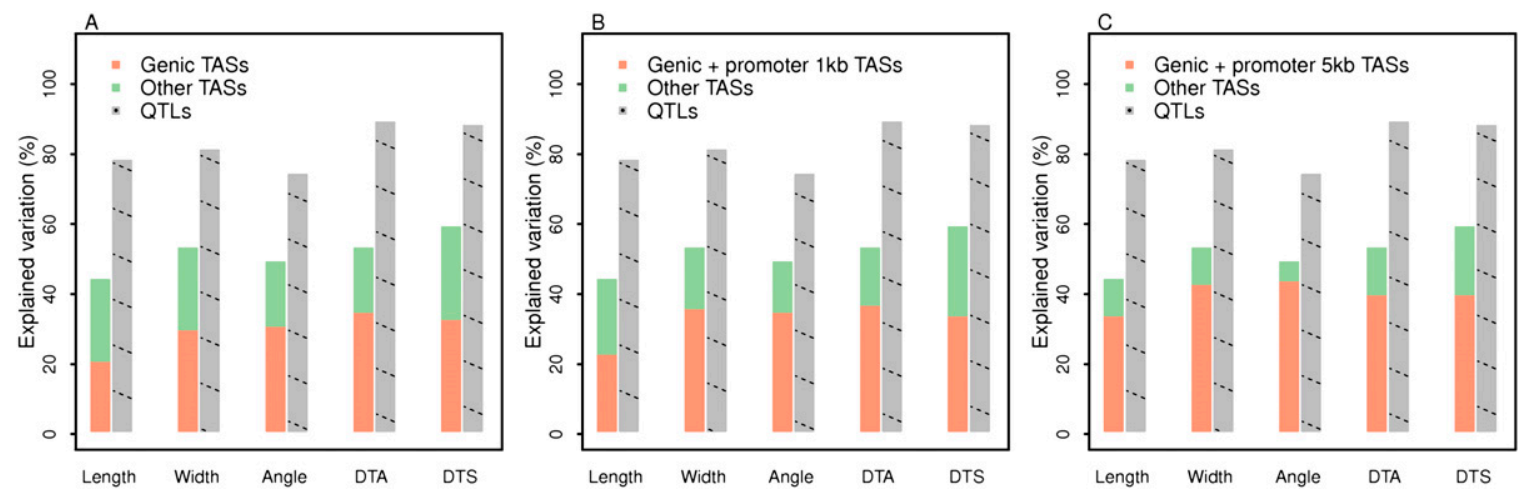

Figure 4. Phenotypic variation explained by genic and nongenic TASs. ( $A$ ) Genic TASs versus all other TASs; $(B)$ genic region plus promoter $1 \mathrm{~kb}$ versus all other TASs; and $(C)$ genic region plus promoter $5 \mathrm{~kb}$ versus all other TASs. Phenotypic variation explained by all QTLs is shown for comparison. TASs located within genic region plus upstream $5 \mathrm{~kb}$ comprise only $13 \%$ of the maize genome but explain a large proportion (67\%-91\%) of the phenotypic variation captured by all TASs.

onstrated the functional outcomes of synonymous mutations (Plotkin and Kudla 2011; Waldman et al. 2011). Tabulation of cloned rice QTLs, which often have larger effects in the respective populations than the TASs identified in the current study, also indicated the importance of expression differences (Miura et al. 2011). In addition, no splicing site mutation or premature stop was found to be TASs. Because the NAM founders were selected to represent a broad range of genetic diversity, these results suggest that in maize, alterations in protein sequence were quantitatively less important during evolution and selection in defining the natural variation in quantitative traits than changes in gene regulation, even though this type of protein sequence change muta-
A Length

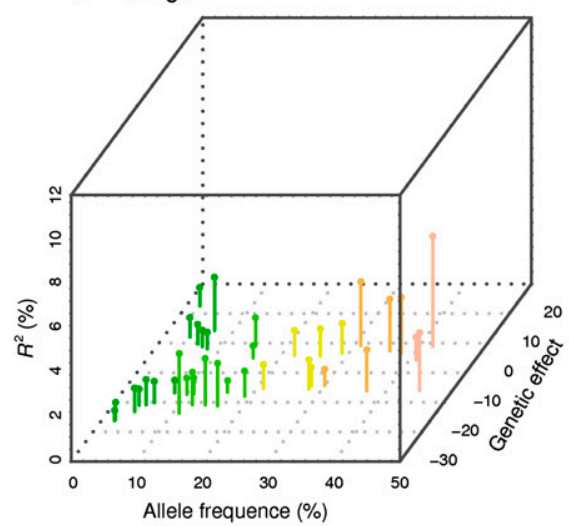

D DTA

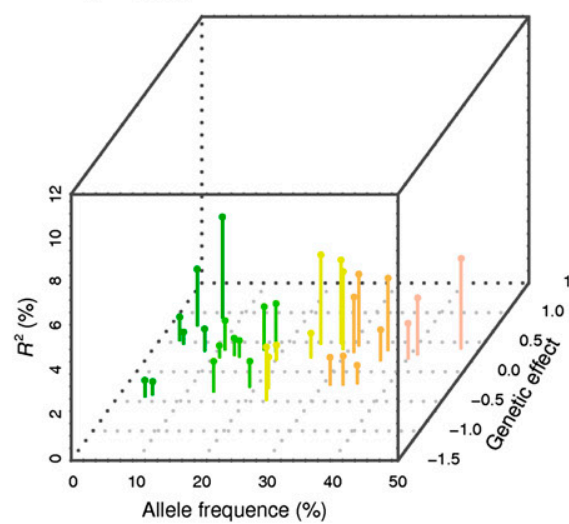

B Width

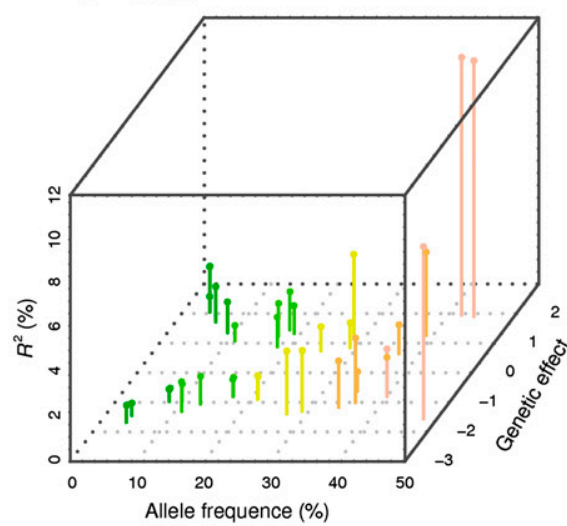

E DTS

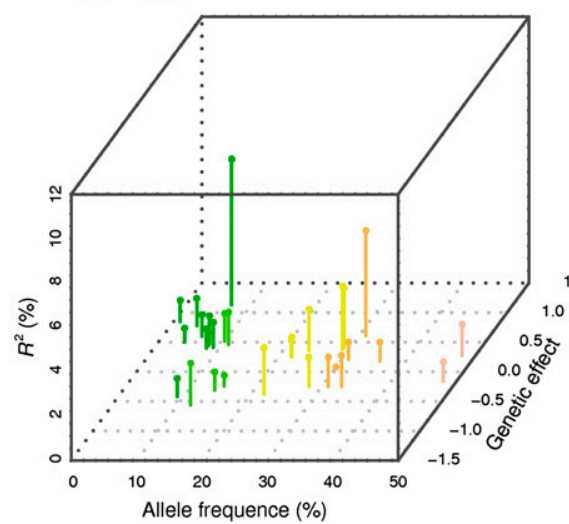

C Angle

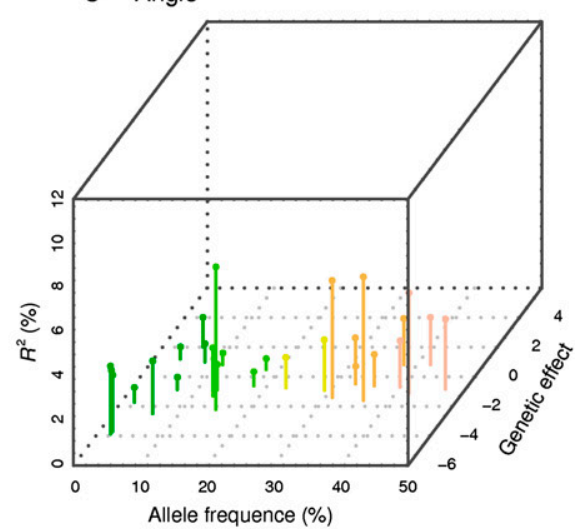

F All traits

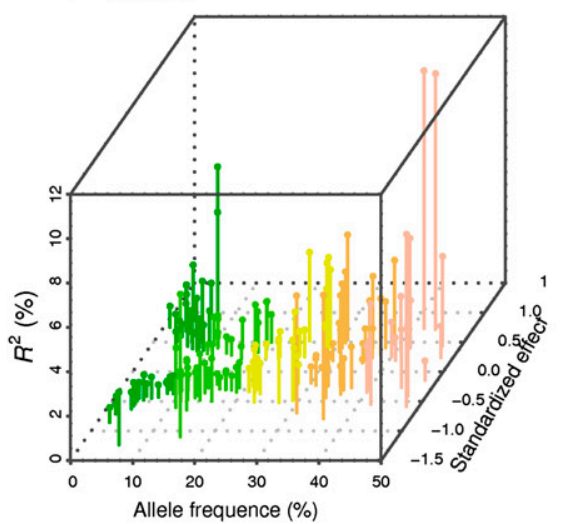

Figure 5. Contribution from an individual TAS to the phenotypic variation is determined by allele frequency and genetic effect size. ( $A$ ) Leaf length in millimeters; $(B)$ leaf width in millimeters; $(C)$ leaf angle in degrees; $(D)$ days to anthesis (DTA); $(E)$ days to silking (DTS); and $(F)$ combined results for all traits with standardized genetic effects. 
tion is often found in genetic studies using individuals with extreme phenotypes. Consistent with this hypothesis, we found that SNPs from promoter regions (5 $\mathrm{kb}$ upstream of the genes) and nongenic region as a whole were overrepresented among TASs (in both the FGS and WGS analyses). These findings imply that longdistance regulatory elements and other forms of causative variants, like structural variations (Lai et al. 2010; Swanson-Wagner et al. 2010), probably also play important roles in the diverse morphology of maize (Stam et al. 2002; Clark et al. 2006; Salvi et al. 2007).

For TAS-implicated candidate genes, the overrepresentation of several GO terms and expression analysis among tissues provided supporting evidence of the dynamic nature of underlying processes of these quantitative traits. Follow-up studies could be prioritized for several of them, which were highlighted with additional support.

\section{Explained variation and GWAS strategy}

As has been observed in the human GWAS (Manolio et al. 2009), maize TASs also captured a portion of phenotypic variation. One potential source of unexplained variation in human diseases could be the action of rare variants having large effects. With the NAM design, the allele frequency captured within the non-B73 founders determines the actual frequency in the population, which affects the phenotypic variation explained. The percentage of variation explained by the TASs with the lowest possible minor allele frequency in the maize NAM population ( $2 \%$, i.e., the case where only one NAM founder has different allele than B73) ranged from $0 \%-9 \%$ for these traits, demonstrating that the contribution of low-frequency alleles varied among quantitative traits (Fig. 5).

Interestingly, although TASs explained less phenotypic variation than QTLs, for all five traits TASs yielded a better model fit than QTLs as indicated by Bayesian information criterion (BIC) values. While QTL analysis in the first stage required many more model parameters to capture multiple alleles, the focus of the genome scan was to identify TASs, all of which were biallelic. Because of a higher penalty associated with the greater number of model parameters, the model fit was better for TASs than QTLs. Future studies may be able to explain more of the phenotypic variation and achieve a better model fit by considering haplotypes and functional predictions of multiple SNPs (Dickson et al. 2010; Singleton et al. 2010).

\section{Efficient GWAS by including promoter regions}

One of the main goals of this study was to evaluate the relative contributions of genic and nongenic polymorphisms to phenotypic variation for quantitative traits. Three relevant findings emerged from this study: (1) approximately half of the dissected regions had genic TASs and the other half had nongenic TASs, (2) genic or nongenic TASs contributed nearly half of phenotypic variation explained by all TASs, and (3) nongenic polymorphisms were significantly overrepresented among TASs, indicating the importance of analyzing nongenic regions in GWAS (Hindorff et al. 2009; Yang et al. 2011). But more importantly, because a significant fraction of the nongenic TASs and their corresponding contributions to the phenotypic variation explained were from the promoter regions upstream of genes (Figs. 3, 4; Supplemental Figs. S10, S11), targeting polymorphisms within genes and their promoter regions deserves attention.
GWAS across a very large number of samples genotyped by whole-genome sequencing would provide the most comprehensive understanding of genetic architecture. The cost of such an approach could, however, be prohibitive, especially for species with large genomes (Cirulli and Goldstein 2010). Our results suggest that at least in maize, the bulk of TASs are located in genes and their promoters. Hence, the combination of RNA-seq and exome capture experiments using long-read (e.g., 454) and paired-end (Illumina and 454) technologies would facilitate the cost-effective identification of promoter and genic polymorphisms for routine GWAS scans in species with complex genomes.

\section{Methods}

\section{Maize population}

The NAM population was developed by crossing 25 diverse founders (the NAM founders) to a common parent B73, whose genome serves as the reference genome for maize (Schnable et al. 2009). Approximately 200 RILs were developed from each cross (McMullen et al. 2009). All founders and 4892 RILs (including 200 RILs from the intermated B73 $\times$ Mo17 population) were genotyped with 1106 tSNPs. A consensus genetic map of the NAM population was constructed based on the tSNPs. The maize HapMap project sequenced genomic DNA from the NAM founders using an Illumina Genome Analyzer and discovered SNPs by aligning the reads to the RefGen_v1 B73 genome (Gore et al. 2009).

\section{Discovery of SNPs via RNA-seq}

Total RNA was extracted from the shoot apex from 2-wk-old seedlings of the NAM founders using TRIzol reagent (Invitrogen). The Poly(A) RNA was isolated from total RNA with oligo d(T) beads and then used to construct the RNA-seq libraries, which were sequenced using the Illumina Genome Analyzer II instrument.

Raw sequence reads were scanned for low-quality bases. Nucleotides with phred quality values less than 15 were trimmed. Each read was examined in two phases. In the first phase, reads were scanned starting at each end, and nucleotides with quality values lower than the threshold were removed. The remaining nucleotides were then scanned using overlapping windows of 10 $\mathrm{bp}$, and sequences beyond the last window with average quality value less than the specified threshold were truncated. Trimmed reads were then aligned to the B73 reference genome (RefGen_v1) using GSNAP (Wu and Nacu 2010), and uniquely mapped reads (two or fewer mismatches every $36 \mathrm{bp}$ and $\leq 3$-bp tails allowed) were used for SNP discovery using the 123SNP software (available at http://schnablelab.plantgenomics.iastate.edu/software/). SNP sites were called only if the site contains at least three reads supporting the base call with error rates $<0.03 \%$, and the most common allele must account for at least $80 \%$ of all aligned reads covering that nucleotide position.

\section{SNP annotation}

SNPs were assigned as genic or nongenic based on their locations relative to two sets of annotated maize genes from maize RefGen_v1. The FGS $(N=32,540)$ includes stringently called genes and is therefore expected to include only a low number of falsepositive calls but to incompletely sample the full gene space. Based on an analysis of an independent RNA-seq data, nearly all (99\%) of the 15,097 genes from the FGS that include SNPs are expressed in maize seedling leaves ( $\mathrm{Li}$ et al. 2010). In contrast, the WGS ( $N=$ $109,563)$ was generated using less stringent parameters and is

\section{Genome Research}


therefore expected to sample more of the gene space but includes more false-positive gene calls. The current estimate of gene number in maize also considers the classical gene set, a collection of $N=$ 464 well-characterized ("true") genes, each of which has been cloned, confers a phenotype when mutated, and is supported by at least three citations (Schnable and Freeling 2011). Approximately two-thirds of the classical gene set is included within the FGS, suggesting that the true gene number in maize is about 50,000 $(3 / 2 \times 32,540)$. The entire classical gene set is included in the WGS, suggesting that the WGS contains most of the gene space.

For genes with alternative splicing isoforms, the transcript with the longest coding region was selected. The functional effects of SNPs (synonymous mutation, nonsynonymous mutation, splice site mutation, premature stop, or frame shift) were annotated using custom perl scripts.

\section{Statistical analysis}

To avoid over-prediction, SNPs for which genotyping data were missing for more than five of the NAM founders were not included in the association analysis. Other missing genotypes were imputed by fastPHASE 1.2 using default parameters (Scheet and Stephens 2006). The imputed SNPs between two adjacent informative tSNPs were projected from founders to RILs based on information from tSNPs (Tian et al. 2011).

The phenotypic data were available at the Panzea database (www.panzea.org). In brief, three leaf traits (leaf length, leaf width, and upper leaf angle) and two flowering times (days to anthesis and days to silking) were collected in eight summer environments across 2 yr. The best linear unbiased predictor (BLUP) of each line was used for linkage and association analysis. Joint linkage analysis across BLUP phenotype and 1106 of tSNPs was conducted in SAS 9.1 with the GLMSelect Procedure. The parameters (population effect and tSNP by population effect) were estimated. tSNPs that met the significant $P$-value $\left(7.1 \times 10^{-5}\right)$ of the marginal F-test in the final model were identified as QTLs.

GWAS was conducted in R (www.r-project.org). To control for polygenic background effects, we conducted the scan by including other QTLs located $10 \mathrm{cM}$ away from the testing region (Supplemental Fig. S4). A dissected region was bordered by three flanking tSNPs from each side of a QTL tSNP (Supplemental Fig. S4). Each dissected region was classified as either predominantly genic if it contains a higher proportion of genic than nongenic SNPs, or predominantly nongenic if otherwise. The SNP with the highest association value was retrieved from each dissected QTL region and designated as the TAS. In cases where multiple TASs were tied in association signal strength, these TASs were individually classified, and the ratio of genic:nongenic TASs determined the final classification (Fig. 2; Supplemental Fig. S2). Test of independence was conducted between the classifications of TASs and the target regions. To address multiple testing issues, the $Q$-value (i.e., adjusted $P$-value by adopting a false-discovery rate controlling procedure) was estimated with $Q$-value (Storey and Tibshirani 2003).

The likelihood-ratio-based $R^{2}$ was used for calculating the phenotypic variation explained by all QTLs and the same procedure for all TASs (Sun et al. 2010). The contributions from genic and nongenic TASs were calculated based on sum of squares from the combined model with both types of TASs fitted, and the same procedure for the contribution from TASs with the lowest minorallele-frequency.

We mapped the SNPs (including TASs) into eight annotation sets (nongenic region, promoter $5 \mathrm{~kb}$, promoter $1 \mathrm{~kb}$, intron, $3^{\prime}$ UTR, 5' UTR, synonymous site, and nonsynonymous site). The nongenic region included promoter $5 \mathrm{~kb}$, which was defined as from the upstream $5 \mathrm{~kb}$ to the TSS. Similarly, promoter $1 \mathrm{~kb}$ covered the region from the upstream $1 \mathrm{~kb}$ to the TSS. To avoid the overlap issue, the TAS distribution among different annotation used only the data from 114 QTL regions with unique TASs. The proportion of TASs in each annotation set was tested for enrichment or depletion against the corresponding proportion of SNPs.

\section{Candidate gene}

GO annotations of the filtered gene set from maize RefGen_v1 were obtained from MaizeSequence (www.maizesequence. org). Overrepresentation of the GO terms with more than one TAS-implicated gene was tested by comparing the proportion of the specific GO term among all implicated genes against all annotated maize genes. The significance of overrepresentation was corrected with the Bonferroni method (Rice 1989).

We sequenced RNA from four developmental stages of the embryo (proembryo, transition phase, coleoptile stage, and L1 stage embryo), and SAMs and lateral meristems (from 2-wk-old seedlings) of B73 (available at www.maizegdb.org). The expression patterns of TAS-implicated candidate genes were also analyzed using previously published transcriptome data from four zones (basal zone, transitional zone, maturing zone, and mature zone) along the proximodistal gradient of maize B73 seedling leaves ( $\mathrm{Li}$ et al. 2010).

\section{Transcriptome analysis of $I b l 1$ mutants}

Whole embryos of $l b l 1$ mutants and wild type (B73 or W22, into which $l b l 1$ had been introgressed) were isolated from developing seeds. mRNA from the embryos was then extracted. RNA-seq was conducted on these samples using an Illumina Genome Analyzer II with three replications. The TAS-implicated candidate genes for leaf length and leaf width were screened to determine whether they were differentially expressed.

\section{Data access}

The RNA-seq data from shoot apex have been deposited in the NCBI Sequence Read Archive (SRA) (http://www.ncbi.nlm.nih. gov/sra) under accession number SRA050451.

\section{Acknowledgments}

This work is supported by the National Science Foundation (DBI-0820610).

Author contributions: J.Y., P.S.S., M.C.P.T., G.J.M, E.S.B., and M.J.S. designed the study. X.L. C.Z., C.-T.Y., W.W., E.M.T., K.A.P, and F.T. performed the analyses. X.L., J.Y., P.S.S., M.J.S., M.C.P.T., G.J.M., and G.B. drafted the manuscript. All of the authors critically revised and provided final approval of this manuscript.

\section{References}

Alonso-Blanco C, Aarts MG, Bentsink L, Keurentjes JJ, Reymond M, Vreugdenhil D, Koornneef M. 2009. What has natural variation taught us about plant development, physiology, and adaptation? Plant Cell 21: 1877-1896.

Ashikari M, Sakakibara H, Lin S, Yamamoto T, Takashi T, Nishimura A, Angeles ER, Qian Q, Kitano H, Matsuoka M. 2005. Cytokinin oxidase regulates rice grain production. Science 309: 741-745.

Atwell S, Huang YS, Vilhjalmsson BJ, Willems G, Horton M, Li Y, Meng D, Platt A, Tarone AM, Hu TT, et al. 2010. Genome-wide association study of 107 phenotypes in Arabidopsis thaliana inbred lines. Nature 465: 627631.

Barbazuk WB, Emrich SJ, Chen HD, Li L, Schnable PS. 2007. SNP discovery via 454 transcriptome sequencing. Plant J 51: 910-918.

Bernardo R, Yu J. 2007. Prospects for genomewide selection for quantitative traits in maize. Crop Sci 47: 1082-1090. 
Buckler ES, Holland JB, Bradbury PJ, Acharya CB, Brown PJ, Browne C, Ersoz E, Flint-Garcia S, Garcia A, Glaubitz JC, et al. 2009. The genetic architecture of maize flowering time. Science 325: 714-718.

Chang BL, Cramer SD, Wiklund F, Isaacs SD, Stevens VL, Sun J, Smith S, Pruett K, Romero LM, Wiley KE, et al. 2009. Fine mapping association study and functional analysis implicate a SNP in MSMB at 10q11 as a causal variant for prostate cancer risk. Hum Mol Genet 18: 1368-1375.

Cirulli ET, Goldstein DB. 2010. Uncovering the roles of rare variants in common disease through whole-genome sequencing. Nat Rev Genet 11 $415-425$.

Cirulli ET, Singh A, Shianna KV, Ge D, Smith JP, Maia JM, Heinzen EL, Goedert JJ, Goldstein DB. 2010. Screening the human exome: A comparison of whole genome and whole transcriptome sequencing. Genome Biol 11: R57. doi: 10.1186/gb-2010-11-5-r57.

Clark RM, Wagler TN, Quijada P, Doebley J. 2006. A distant upstream enhancer at the maize domestication gene $t b 1$ has pleiotropic effects on plant and inflorescent architecture. Nat Genet 38: 594-597.

Cloonan N, Forrest AR, Kolle G, Gardiner BB, Faulkner GJ, Brown MK, Taylor DF, Steptoe AL, Wani S, Bethel G, et al. 2008. Stem cell transcriptome profiling via massive-scale mRNA sequencing. Nat Methods 5: 613-619.

Dickson SP, Wang K, Krantz I, Hakonarson H, Goldstein DB. 2010. Rare variants create synthetic genome-wide associations. PLoS Biol 8: e1000294. doi: 10.1371/journal.pbio.1000294.

Doebley JF, Gaut BS, Smith BD. 2006. The molecular genetics of crop domestication. Cell 127: 1309-1321.

Emrich SJ, Barbazuk WB, Li L, Schnable PS. 2007. Gene discovery and annotation using LCM-454 transcriptome sequencing. Genome Res 17: 69-73.

Frary A, Nesbitt TC, Grandillo S, Knaap E, Cong B, Liu J, Meller J, Elber R, Alpert KB, Tanksley SD. 2000. fw2.2: A quantitative trait locus key to the evolution of tomato fruit size. Science 289: 85-88.

Gore MA, Chia JM, Elshire RJ, Sun Q, Ersoz ES, Hurwitz BL, Peiffer JA, McMullen MD, Grills GS, Ross-Ibarra J, et al. 2009. A first-generation haplotype map of maize. Science 326: 1115-1117.

Harper L, Freeling M. 1996. Interactions of liguleless1 and liguleless2 function during ligule induction in maize. Genetics 144: 1871-1882.

Hindorff LA, Sethupathy P, Junkins HA, Ramos EM, Mehta JP, Collins FS, Manolio TA. 2009. Potential etiologic and functional implications of genome-wide association loci for human diseases and traits. Proc Natl Acad Sci 106: 9362-9367.

Huang X, Wei X, Sang T, Zhao Q, Feng Q, Zhao Y, Li C, Zhu C, Lu T, Zhang Z, et al. 2010. Genome-wide association studies of 14 agronomic traits in rice landraces. Nat Genet 42: 961-967.

Lai J, Li R, Xu X, Jin W, Xu M, Zhao H, Xiang Z, Song W, Ying K, Zhang M, et al. 2010. Genome-wide patterns of genetic variation among elite maize inbred lines. Nat Genet 42: 1027-1030.

Lee JY, Baum SF, Alvarez J, Patel A, Chitwood DH, Bowman JL. 2005. Activation of CRABS CLAW in the nectaries and carpels of Arabidopsis. Plant Cell 17: 25-36.

Li P, Ponnala L, Gandotra N, Wang L, Si Y, Tausta SL, Kebrom TH, Provart N, Patel R, Myers CR, et al. 2010. The developmental dynamics of the maize leaf transcriptome. Nat Genet 42: 1060-1067.

Manolio TA, Collins FS, Cox NJ, Goldstein DB, Hindorff LA, Hunter DJ, McCarthy MI, Ramos EM, Cardon LR, Chakravarti A, et al. 2009. Finding the missing heritability of complex diseases. Nature 461: 747-753.

McMullen MD, Kresovich S, Villeda HS, Bradbury P, Li H, Sun Q, Flint-Garcia S, Thornsberry J, Acharya C, Bottoms C, et al. 2009. Genetic properties of the maize nested association mapping population. Science 325: 737740 .

Mena M, Mandel MA, Lerner DR, Yanofsky MF, Schmidt RJ. 1995. A characterization of the MADS-box gene family in maize. Plant J 8: 845854

Meyer KB, Maia AT, O'Reilly M, Teschendorff AE, Chin SF, Caldas C, Ponder BA. 2008. Allele-specific up-regulation of FGFR2 increases susceptibility to breast cancer. PLoS Biol 6: e108. doi: 10.1371/journal.pbio.0060108.

Miura K, Ashikari M, Matsuoka M. 2011. The role of QTLs in the breeding of high-yielding rice. Trends Plant Sci 16: 319-326.

Nogueira FT, Madi S, Chitwood DH, Juarez MT, Timmermans MC. 2007. Two small regulatory RNAs establish opposing fates of a developmental axis. Genes Dev 21: 750-755.

Plotkin JB, Kudla G. 2011. Synonymous but not the same: The causes and consequences of codon bias. Nat Rev Genet 12: 32-42.

Rice WR. 1989. Analyzing tables of statistical tests. Evolution 43: 223-225.

Riedelsheimer C, Czedik-Eysenberg A, Grieder C, Lisec J, Technow F, Sulpice R, Altmann T, Stitt M, Willmitzer L, Melchinger AE. 2012. Genomic and metabolic prediction of complex heterotic traits in hybrid maize. Nat Genet 44: 217-220.
Salvi S, Sponza G, Morgante M, Tomes D, Niu X, Fengler KA, Meeley R, Ananiev EV, Svitashev S, Bruggemann E, et al. 2007. Conserved noncoding genomic sequences associated with a flowering-time quantitative trait locus in maize. Proc Natl Acad Sci 104: 11376-11381.

Scheet P, Stephens M. 2006. A fast and flexible statistical model for largescale population genotype data: Applications to inferring missing genotypes and haplotypic phase. Am I Hum Genet 78: 629-644.

Schnable JC, Freeling M. 2011. Genes identified by visible mutant phenotypes show increased bias toward one of two subgenomes of maize. PLOS ONE 6: e17855. doi: 10.1371/journal.pone.0017855.

Schnable PS, Ware D, Fulton RS, Stein JC, Wei F, Pasternak S, Liang C, Zhang J, Fulton L, Graves TA, et al. 2009. The B73 maize genome: Complexity, diversity, and dynamics. Science 326: 1112-1115.

Schnable JC, Springer NM, Freeling M. 2011. Differentiation of the maize subgenomes by genome dominance and both ancient and ongoing gene loss. Proc Natl Acad Sci 108: 4069-4074.

Schneeberger RG, Becraft PW, Hake S, Freeling M. 1995. Ectopic expression of the knox homeo box gene rough sheath1 alters cell fate in the maize leaf. Genes Dev 9: 2292-2304.

Sekhon RS, Lin H, Childs KL, Hansey CN, Buell CR, de Leon N, Kaeppler SM 2011. Genome-wide atlas of transcription during maize development. Plant J 66: 553-563.

Singleton AB, Hardy J, Traynor BJ, Houlden H. 2010. Towards a complete resolution of the genetic architecture of disease. Trends Genet 26: 438442.

Stam M, Belele C, Ramakrishna W, Dorweiler JE, Bennetzen JL, Chandler VL. 2002. The regulatory regions required for $B^{\prime}$ paramutation and expression are located far upstream of the maize $b 1$ transcribed sequences. Genetics 162: 917-930.

Stenson PD, Mort M, Ball EV, Howells K, Phillips AD, Thomas NS, Cooper DN. 2009. The Human Gene Mutation Database: 2008 update. Genome Med 1: 13. doi: $10.1186 / \mathrm{gm} 13$.

Storey JD, Tibshirani R. 2003. Statistical significance for genomewide studies. Proc Natl Acad Sci 100: 9440-9445.

Sun G, Zhu C, Kramer MH, Yang SS, Song W, Piepho HP, Yu J. 2010. Variation explained in mixed-model association mapping. Heredity 105: 333-340.

Swanson-Wagner RA, Eichten SR, Kumari S, Tiffin P, Stein JC, Ware D, Springer NM. 2010. Pervasive gene content variation and copy number variation in maize and its undomesticated progenitor. Genome Res 20: 1689-1699.

Teslovich TM, Musunuru K, Smith AV, Edmondson AC, Stylianou IM, Koseki M, Pirruccello JP, Ripatti S, Chasman DI, Willer CJ, et al. 2010. Biological, clinical and population relevance of 95 loci for blood lipids. Nature 466: 707-713.

Tian F, Bradbury PJ, Brown PJ, Hung H, Sun Q, Flint-Garcia S, Rocheford TR, McMullen MD, Holland JB, Buckler ES. 2011. Genome-wide association study of leaf architecture in the maize nested association mapping population. Nat Genet 43: 159-162.

Timmermans MC, Schultes NP, Jankovsky JP, Nelson T. 1998. Leafbladeless1 is required for dorsoventrality of lateral organs in maize. Development 125: $2813-2823$.

Waldman YY, Tuller T, Keinan A, Ruppin E. 2011. Selection for translation efficiency on synonymous polymorphisms in recent human evolution. Genome Biol Evol 3: 749-761.

Walsh J, Waters CA, Freeling M. 1998. The maize gene liguleless2 encodes a basic leucine zipper protein involved in the establishment of the leaf blade-sheath boundary. Genes Dev 12: 208-218.

Wu TD, Nacu S. 2010. Fast and SNP-tolerant detection of complex variants and splicing in short reads. Bioinformatics 26: $873-881$.

Yang J, Manolio TA, Pasquale LR, Boerwinkle E, Caporaso N, Cunningham JM, de Andrade M, Feenstra B, Feingold E, Hayes MG, et al. 2011. Genome partitioning of genetic variation for complex traits using common SNPs. Nat Genet 43: 519-525.

Yu J, Pressoir G, Briggs WH, Vroh Bi I, Yamasaki M, Doebley JF, McMullen MD, Gaut BS, Nielsen DM, Holland JB, et al. 2006. A unified mixedmodel method for association mapping that accounts for multiple levels of relatedness. Nat Genet 38: 203-208.

Yu J, Holland JB, McMullen MD, Buckler ES. 2008. Genetic design and statistical power of nested association mapping in maize. Genetics 178: $539-551$.

Zhang Z, Ersoz E, Lai CQ, Todhunter RJ, Tiwari HK, Gore MA, Bradbury PJ, Yu J, Arnett DK, Ordovas JM, et al. 2010. Mixed linear model approach adapted for genome-wide association studies. Nat Genet 42: 355-360.

Received March 7, 2012; accepted in revised form June 7, 2012.

\section{Genome Research}

www.genome.org 


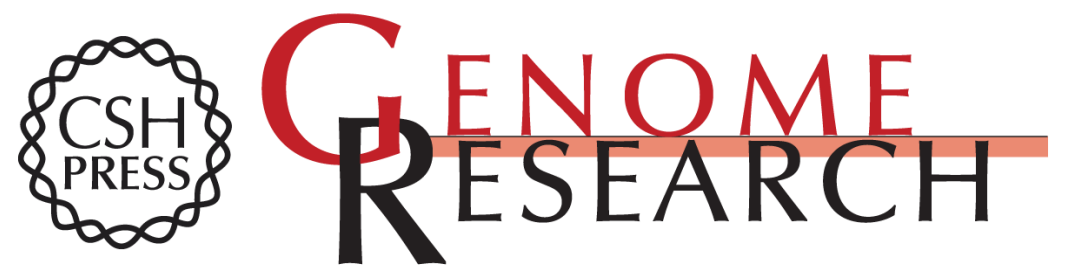

\section{Genic and nongenic contributions to natural variation of quantitative traits in maize}

Xianran Li, Chengsong Zhu, Cheng-Ting Yeh, et al.

Genome Res. 2012 22: 2436-2444 originally published online June 14, 2012

Access the most recent version at doi:10.1101/gr.140277.112

Supplemental Material

References

Open Access

Creative Commons

License

Email Alerting
http://genome.cshlp.org/content/suppl/2012/09/26/gr.140277.112.DC1

This article cites 54 articles, 18 of which can be accessed free at: http://genome.cshlp.org/content/22/12/2436.full.html\#ref-list-1

Freely available online through the Genome Research Open Access option.

This article is distributed exclusively by Cold Spring Harbor Laboratory Press for the first six months after the full-issue publication date (see

http://genome.cshlp.org/site/misc/terms.xhtml). After six months, it is available under a Creative Commons License (Attribution-NonCommercial 3.0 Unported License), as described at http://creativecommons.org/licenses/by-nc/3.0/.

Receive free email alerts when new articles cite this article - sign up in the box at the top right corner of the article or click here.

\section{Affordable, Accurate Sequencing.}

To subscribe to Genome Research go to:

https://genome.cshlp.org/subscriptions 